\title{
Early consciousness disorder in acute ischemic stroke: incidence, risk factors and outcome
}

\author{
Jie Li ${ }^{1,2+}$, Deren Wang ${ }^{1+} \mathbb{B}$, Wendan Tao ${ }^{1}$, Wei Dong ${ }^{1}$, Jing Zhang ${ }^{1}$, Jie Yang ${ }^{3}$ and Ming Liu $^{1 *}$
}

\begin{abstract}
Background: Little is known about the incidence and risk factors of early consciousness disorder (ECD) in patients with acute ischemic stroke, or about how ECD may affect complications and outcomes.

Methods: Patients admitted to our hospital within $24 \mathrm{~h}$ of onset of acute ischemic stroke were consecutively enrolled. ECD was evaluated clinically and using the Glasgow coma scale. Multivariate analysis was used to identify risk factors of ECD, as well as associations between ECD and clinical outcomes.

Results: Of the 569 patients enrolled, 199 (35\%) had ECD. Independent risk factors of ECD were advanced age (OR 1. 027, $95 \%$ Cl 1.007 to 1.048), National Institutes of Health Stroke Score on admission (OR 1.331, 95 \% Cl 1.257 to 1.410), and massive cerebral infarct (OR 3.211, $95 \% \mathrm{Cl} 1.642$ to 6.279). ECD was associated with higher frequency of strokerelated complications ( $83.4 \%$ vs. $31.1 \%, P<0.001)$ and higher in-hospital mortality $(17.1 \%$ vs. $0.5 \%, P<0.001)$. ECD independently predicted 3-month death/disability (OR 3.272, $95 \% \mathrm{Cl} 1.670$ to 6.413).
\end{abstract}

Conclusions: ECD is prevalent in Chinese patients with acute ischemic stroke. Risk factors include advanced age, stroke severity, and massive cerebral infarct. ECD is associated with higher frequency of stroke-related complications and 3-month death/disability.

Keywords: Ischemic stroke, Early consciousness disorder, Occurrence, Risk factor, Complication, Outcome

Abbreviations: ECD, Early consciousness disorder; CT, Computed tomography; MRI, Magnetic resonance imaging; mRS, Modified Rankin Scale; NIHSS, National Institutes of Health Stroke Scale; GCS, Glasgow coma scale; ORs, Odds ratios; $95 \%$ Cls, $95 \%$ confidence intervals

\section{Background}

Stroke is the second most frequent cause of death and the most frequent cause of disability worldwide. In China, stroke has become the leading cause of death and disability in both urban and rural populations, with incidence increasing every year [1]. Approximately $80 \%$ of all strokes are ischemic [1].

Consciousness, which refers to awareness of self and environment, depends on levels of arousal and wakefulness, as well as stimulus content [2]. Many patients in early stages of acute ischemic stroke show acute disorder

\footnotetext{
* Correspondence: wyplmh@hotmail.com

${ }^{\dagger}$ Equal contributors

'Department of Neurology, Stroke Clinical Research Unit, West China Hospital, Sichuan University, No. 37, GuoXue Xiang, Chengdu 610041, Sichuan, People's Republic of China

Full list of author information is available at the end of the article
}

of consciousness, a condition known as early consciousness disorder (ECD). In fact, stroke is one of the three most frequent causes of conscious disturbance in emergency rooms, together with trauma and hypoglycemia [3]. Data from various stroke registries suggest that $4-38 \%$ of stroke patients experience decreased level of consciousness or coma, and 13-48 \% experience confusion or delirium [4-11].

Little is known about the incidence or risk factors of ECD in patients who experience acute ischemic stroke. This is an important question, because early consciousness disorder may disturb physician' history-taking in clinical practice and ECD in the acute stroke period may predict stroke progression and increase risk of strokeassociated pneumonia [12-14]. Studies have found several possible risk factors of acute disorder of 
consciousness after stroke: age, sex, previous stroke, atrial fibrillation, diabetes mellitus, alcohol consumption, stroke severity, infarct sites and, according to some reports, massive cerebral infarct and multiple brain infarcts [15-19]. However, currently available information are rough data extracted from registries for different population. Direct comparative data between stroke patients with ECD and without ECD especially on prospective study are scarce. Further work is needed to identify a reliable set of ECD predictors.

Also unclear is whether ECD influences stroke-related complications and clinical outcomes in stroke patients. This is made more important by the fact that the presence of ECD in a stroke patient can affect decisions about treatment and management.

To address these questions, the present prospective study analyzed potential ECD risk factors and associated outcomes in a mid-sized cohort of Chinese patients with acute ischemic stroke.

\section{Methods \\ Subjects}

Between 1 January 2009 and 31 December 2010, patients with first-ever or recurrent stroke who were admitted to the Department of Neurology at West China Hospital of Sichuan University were consecutively and prospectively registered into the Chengdu Stroke Registry database as described [20]. We enrolled into the present study only those patients who were admitted within $24 \mathrm{~h}$ of symptom onset and whose diagnosis of ischemic stroke was confirmed by computed tomography $(\mathrm{CT})$ or magnetic resonance imaging (MRI) of the brain. We excluded patients who had previously received a modified Rankin Scale (mRS) score $>2$ or who had premorbid conditions such as trauma, intoxication, infection, metabolic or other systemic disease.

\section{Baseline assessment}

Using a standardized form, we collected patient data at baseline that included age, sex, time between stroke onset and admission, initial stroke severity [assessed using the National Institutes of Health Stroke Scale (NIHSS) score], systolic and diastolic blood pressure, serum glucose concentration, Glasgow coma scale (GCS) score, stroke risk factors and results of neurological imaging. Stroke risk factors in the present study included hypertension, diabetes mellitus, hyperlipidemia, coronary heart disease, atrial fibrillation, valvular heart disease, previous stroke, history of head trauma, current smoking and alcohol consumption [20]. Neurological imaging findings analyzed in this study were where infarcts occurred (frontal, parietal, temporal, occipital and insular lobe, basal ganglia, subcortical white matter, thalamus, brain stem, cerebellum), whether the left or right hemisphere was involved, and whether the posterior or anterior circulation was involved [21,22].

Patients in which CT or MRI revealed involvement of more than half the middle cerebral artery distribution were diagnosed as having massive cerebral infarct [23]. Multiple brain infarction was defined as multiple recent infarcts involving non-contiguous regions of abnormality across multiple vascular territories [24]. TOAST criteria were used to assign the likely stroke etiology in each patient as large-artery atherosclerosis, cardioembolism, small-artery occlusion, other determined etiology or undetermined etiology [25].

Conscious state at baseline (initially when admitted to the neurology ward) was independently assessed by two experienced neurologists according to Adams and Victor's Principles of Neurology (9th edition) [26], via evaluating the patient's wakefulness, verbal and motor response, orientation to person, place and time, and other physical examination. Patients classified as impaired consciousness (Somnolence, Stupor, Coma, Confusion or Delirium) were assigned to the ECD group, while others were assigned to the no-ECD group.

\section{In-hospital and post-discharge outcomes}

Hospital staff determined in-hospital stroke-related complications and mortality based on record review after patient discharge or death. At 3 months after stroke onset, patients were contacted for follow-up and asked to fill out a questionnaire during a structured telephone interview or by post. The primary outcomes in this study were in-hospital death and either death or disability during the 3-month follow-up. Disability was defined as an mRS score of 3-5 [27].

\section{Statistical analysis}

All statistical analyses were performed using SPSS for Windows 16.0 (IBM, Chicago, IL, USA). Inter-group differences for continuous variables were assessed for significance using Student's $t$-test or the Mann-Whitney $U$ test, while differences for categorical variables were assessed using the chi-squared and Fisher exact tests. Multivariate logistic regression by a backward stepwise procedure was used to identify independent risk factors of ECD on admission. Variables were eliminated from the model if their associated $\mathrm{P}$ was $>0.10$. Logistic regression was used to identify independent predictors of 3-month death/disability. When appropriate, effect sizes were estimated using odds ratios (ORs) and $95 \%$ confidence intervals (95\% CIs). All significance levels mentioned are 2-tailed, and the significance threshold was defined as $P<0.05$.

\section{Results}

Between 1 January 2009 and 31 December 2010, 1,506 patients with acute ischemic stroke were consecutively 
and prospectively registered in the Chengdu Stroke Registry. Of those, 569 (37.8 \%) were admitted to our hospital within $24 \mathrm{~h}$ of stroke onset and were therefore included in the present study. Of this group, comprising 303 men and 266 women aged $64.75 \pm 14.02$ years, 199 (35\%) had ECD on admission, of whom 38 (6.7 \%) were Coma. Among the 143 patients in our cohort with hyper-acute ischemic stroke who were admitted within 6 $\mathrm{h}$ of symptom onset, ECD occurred in 52 (36.4\%). Among the 81 patients admitted within $4.5 \mathrm{~h}$ of symptom onset, ECD occurred in 29 (35.8\%).

\section{ECD and baseline characteristics}

Comparison of baseline characteristics of patients with or without ECD (Table 1) showed that those with ECD were older, had higher serum glucose concentrations, higher median NIHSS score and lower median GCS score. Patients with ECD also showed higher rates of several stroke risk factors (coronary heart disease, atrial

Table 1 Baseline characteristics of Chinese patients with acute ischemic stroke and with or without early consciousness disorder

\begin{tabular}{|c|c|c|c|}
\hline & With ECD & Without ECD & $P$ \\
\hline Patients, n (\%) & $199(35.0)$ & $370(65.0)$ & - - \\
\hline Mean age, yr & $66.7 \pm 13.6$ & $63.7 \pm 14.2$ & $0.016^{*}$ \\
\hline Women, n (\%) & $103(51.8)$ & $163(44.1)$ & $0.079^{* * *}$ \\
\hline $\begin{array}{l}\text { Delay from symptom onset to } \\
\text { admission, } h\end{array}$ & $13.9 \pm 8.2$ & $15.0 \pm 8.5$ & $0.130^{*}$ \\
\hline $\begin{array}{l}\text { Median NIHSS score on } \\
\text { admission }\end{array}$ & $16(0-33)$ & $3.5(0-19)$ & $<0.001^{* *}$ \\
\hline Median GCS score on admission & $10(3-15)$ & $15(9-15)$ & $<0.001^{* *}$ \\
\hline $\mathrm{SBP} \geq 180 \mathrm{mmHg}, \mathrm{n}(\%)$ & $16(8.0)$ & $24(6.5)$ & $0.489^{* * *}$ \\
\hline DBP $\geq 100$ mmHg, n (\%) & $32(16.1)$ & $43(11.6)$ & $0.134^{* * *}$ \\
\hline $\begin{array}{l}\text { Serum glucose on admission, } \\
\mathrm{mmol} / \mathrm{L}\end{array}$ & $8.53 \pm 3.62$ & $7.21 \pm 2.85$ & $<0.001^{*}$ \\
\hline \multicolumn{4}{|l|}{ Stroke risk factors, n (\%) } \\
\hline Hypertension & $119(59.8)$ & $237(64.1)$ & $0.317^{* * *}$ \\
\hline Diabetes mellitus & $56(28.1)$ & $90(24.3)$ & $0.320^{* * *}$ \\
\hline Hyperlipidemia & $34(17.1)$ & $97(26.2)$ & $0.014^{* * *}$ \\
\hline Coronary heart disease & $29(14.6)$ & $28(7.6)$ & $0.008^{* * *}$ \\
\hline Atrial fibrillation & $85(42.7)$ & 70 (18.9) & $<0.001^{* * *}$ \\
\hline Valvular heart disease & 39 (19.6) & $35(9.5)$ & $0.001^{* * *}$ \\
\hline Current smoking & $43(21.6)$ & $101(27.3)$ & $0.137^{* * *}$ \\
\hline Alcohol consumption & $38(19.1)$ & $57(15.4)$ & $0.260^{* * *}$ \\
\hline Previous stroke & $34(17.1)$ & 77 (20.8) & $0.285^{* * *}$ \\
\hline History of head trauma & $2(1.0)$ & $5(1.4)$ & $1.000^{* * *}$ \\
\hline
\end{tabular}

Abbreviations: $D B P$ diastolic blood pressure, ECD early consciousness disorder, GCS Glasgow coma scale, NIHSS National Institutes of Health Stroke Scale, $S B P$ systolic blood pressure

*Student's $t$ test

**Mann-Whitney $U$ test

*** $x^{2}$ test fibrillation and valvular heart disease), but a lower rate of hyperlipidemia.

All patients in the cohort underwent neuro-imaging by CT $(483,84.9 \%)$, MRI $(489,85.9 \%)$ or both (405, $71.2 \%)$. Comparison of imaging findings between patients with or without ECD (Table 2) showed that those with ECD had significantly higher rates of cerebral cortex infarction, midbrain infarction, massive cerebral infarction and anterior circulation involvement.

Patients with ECD showed lower rates of subcortical white matter infarction, thalamus infarction and posterior circulation involvement. The two patient groups did not differ significantly in rates of multiple brain infarction or involvement of the left or right hemispheres.

The distribution of four conscious states differed significantly among the TOAST stroke subtypes $(P<0.001)$. The subtype with the highest incidence of ECD was cardioembolism $(56.1 \%)$, followed by stroke of other determined etiology (52.3\%), large-artery atherosclerosis (39.6\%), stroke of undetermined etiology (33.0\%), and small-artery occlusion (11.0\%).

\section{Risk factors of ECD on admission}

Multivariable logistic regression identified several independent risk factors of ECD in acute ischemic stroke

Table 2 Neurological imaging findings in Chinese patients with acute ischemic stroke and with or without early consciousness disorder

\begin{tabular}{llll}
\hline & With ECD & Without ECD & $P$ \\
\hline Infarct site, $\mathrm{n}(\%)$ & & & \\
Frontal lobe & $115(57.8)$ & $79(21.4)$ & $<0.001$ \\
Parietal lobe & $119(59.8)$ & $90(24.3)$ & $<0.001$ \\
Temporal lobe & $133(66.8)$ & $89(24.1)$ & $<0.001$ \\
Occipital lobe & $56(28.1)$ & $37(10.0)$ & $<0.001$ \\
Insula lobe & $22(11.1)$ & $22(5.9)$ & 0.030 \\
Subcortical white matter & $35(17.6)$ & $100(27.0)$ & 0.012 \\
Basal ganglia & $92(46.2)$ & $184(49.7)$ & 0.408 \\
Thalamus & $15(7.5)$ & $51(13.8)$ & 0.026 \\
Cerebellum & $22(11.1)$ & $24(6.5)$ & 0.057 \\
Midbrain & $11(5.5)$ & $7(1.9)$ & 0.018 \\
Pons & $16(8.0)$ & $47(12.7)$ & 0.091 \\
Medulla & $2(1.0)$ & $13(3.5)$ & 0.075 \\
Massive cerebral infarction, $\mathrm{n}(\%)$ & $124(62.3)$ & $37(10.0)$ & $<0.001$ \\
Multiple brain infarction, $\mathrm{n}(\%)$ & $61(30.7)$ & $136(36.8)$ & 0.144 \\
AC involved, $\mathrm{n}(\%)$ & $172(86.4)$ & $281(75.9)$ & 0.003 \\
PC involved, $\mathrm{n}$ (\%) & $43(21.6)$ & $131(35.4)$ & 0.001 \\
Left hemisphere involved, $\mathrm{n}(\%)$ & $109(54.8)$ & $196(53.0)$ & 0.681 \\
Right hemisphere involved, $\mathrm{n}(\%)$ & $103(51.8)$ & $188(50.8)$ & 0.829 \\
\hline A & 196 (1) & \\
\hline
\end{tabular}

Abbreviations: $A C$ anterior circulation, $P C$ posterior circulation $x^{2}$ test 
patients admitted within $24 \mathrm{~h}$ of stroke onset (Table 3): age (adjusted OR 1.027, $95 \%$ CI 1.007 to 1.048), NIHSS score on admission (adjusted OR 1.331, 95 \% CI 1.257 to 1.410), massive cerebral infarction (adjusted OR 3.211, $95 \%$ CI 1.642 to 6.279), high serum glucose on admission (adjusted OR 1.141, $95 \%$ CI 1.055 to 1.235), and history of alcohol consumption (adjusted OR 2.123, $95 \%$ CI 1.030 to 4.375). Conversely, high systolic pressure on admission appeared to protect against ECD within $24 \mathrm{~h}$ of stroke onset (adjusted OR 0.983, 95 \% CI 0.968 to 0.999 ). Anterior circulation involvement did not independently predict ECD (adjusted OR 1.17, $95 \% \mathrm{CI}$ 0.560 to $2.441, P=0.677$ ).

\section{Stroke-related complications}

The rate of stroke-related complications (excluding stroke recurrence) was higher among patients with ECD than among patients without ECD $(83.4 \%$ vs. $31.1 \%, P<$ 0.001 ; Table 4). The most frequent neurological complication in patients with ECD was malignant edema (30.2\%), followed by hemorrhagic transformation $(22.1 \%)$ and post-stroke epilepsy (11.1\%). Pulmonary infection was the most frequent medical complication among patients with $\operatorname{ECD}(58.3 \%)$.

\section{Outcomes}

Of the 569 patients in our cohort, 29 (5.1\%) were lost during 3-month follow-up, of whom 13 had ECD on admission and 16 did not $(P=0.253)$. Of the remaining 540 patients, those with ECD showed significantly higher rates of in-hospital mortality $(17.1 \%$ vs. $0.5 \%, P<0.001)$, death during 3-month follow-up (33.9 \% vs. $3.1 \%, P<$ 0.001 ) and death/disability (mRS 3-5) during follow-up (78.0 \% vs. $21.8 \%, P<0.001)$. Multivariate logistic regression in which data were adjusted for age, sex and stroke risk factors identified age, GCS score and ECD as independent predictors of death and death/disability at 3 months (Table 5). When NIHSS score and stroke-related complications were included as inputs in this multivariate model, ECD was still the independent predictor of death/ disability at 3 months (adjusted OR 3.272, $95 \%$ CI 1.670

Table 3 Multivariate logistic regression to identify risk factors for early consciousness disorder on admission in Chinese patients with acute ischemic stroke

\begin{tabular}{ll}
\hline Variable & Adjusted $\mathrm{OR}^{\mathrm{a}}(95 \% \mathrm{Cl})$ \\
\hline Age & $1.027(1.007$ to 1.048$)$ \\
NIHSS on admission & $1.331(1.257$ to 1.410$)$ \\
Serum glucose on admission & $1.141(1.055$ to 1.235$)$ \\
Massive cerebral infarction & $3.211(1.642$ to 6.279$)$ \\
History of alcohol consumption & $2.123(1.030$ to 4.375$)$ \\
High systolic pressure & $0.983(0.968$ to 0.999$)$ \\
\hline
\end{tabular}

${ }^{2}$ Adjusted for age, sex, stroke risk factors, stroke severity and imaging findings
Table 4 In-hospital, stroke-related complications in Chinese patients with acute ischemic stroke and with or without early consciousness disorder

\begin{tabular}{llll}
\hline & With ECD & Without ECD & $P$ \\
\hline All complications, n (\%) & $166(83.4)$ & $115(31.1)$ & $<0.001$ \\
Neurological complications, n (\%) & & & \\
Brain oedema & $60(30.2)$ & $6(1.6)$ & $<0.001$ \\
Haemorrhagic transformation & $44(22.1)$ & $26(7.0)$ & $<0.001$ \\
Seizures and epilepsy & $22(11.1)$ & $6(0.6)$ & $<0.001$ \\
Central hyperthermia & $7(3.5)$ & $1(0.3)$ & 0.006 \\
Recurrent stroke & $3(1.5)$ & $3(0.8)$ & 0.730 \\
Medical complications, n (\%) & & & \\
Pulmonary infection & $116(58.3)$ & $64(17.3)$ & $<0.001$ \\
Urinary tract infection & $18(9.0)$ & $10(2.7)$ & 0.001 \\
Gastrointestinal bleeding & $34(17.1)$ & $17(4.6)$ & $<0.001$ \\
Electrolyte disturbance & $81(40.7)$ & $49(13.2)$ & $<0.001$ \\
Acute renal failure & $17(8.5)$ & $6(1.6)$ & $<0.001$ \\
Urinary incontinence & $49(24.6)$ & $12(3.2)$ & $<0.001$ \\
Bed sores & $10(5.0)$ & $3(0.8)$ & 0.004 \\
Phlebothrombosis & $6(3.0)$ & $1(0.3)$ & 0.015 \\
Falls & $5(2.5)$ & $0(0)$ & 0.010 \\
\hline$x^{2}$ test & & &
\end{tabular}

to 6.413), but was not the independent predictor of death at 3 months (adjusted OR 1.641, $95 \%$ CI 0.749 to 3.592 ).

\section{Discussion}

Although a sizeable proportion of stroke patients are admitted to the neurology ward with conscious disturbance, the incidence and risk factors of ECD are poorly understood, as are potential relationships between ECD and clinical outcomes in acute ischemic stroke. Here we provide evidence that ECD is common in Chinese patients with acute ischemic stroke, occurring in more than one of every three patients in our cohort that was admitted within $24 \mathrm{~h}$ of symptom onset. In this subset of patients who had ECD, nearly a third was in a state of decreased consciousness or coma on admission. This incidence is within the range of $4.3-37.7 \%$ reported by other stroke registries [4-9]. In contrast, the $5.3 \%$ incidence of delirium in our study is lower than the range of $13-48 \%$ reported for other ethnic groups $[10,11]$. Differences in the incidence of consciousness disorder between our study and others may easily arise from heterogeneity in the time between stroke onset and hospital admission, which most studies in the literature did not strictly control. Such heterogeneity increases the risk that pre-hospital care, disease course and strokerelated complications contributed to observed conscious disturbance. Another simple explanation for differences between our study and others is that ours involved a single 
Table $\mathbf{5}$ In-hospital and 3-month outcomes of Chinese patients with acute ischemic stroke and with or without early consciousness disorder

\begin{tabular}{llllll}
\hline & With ECD & Without ECD & OR (95 \% Cl) & $P$ & Adjusted OR (95 \% Cl) \\
\hline In-hospital death, $\mathrm{n}(\%)$ & $34(17.1)$ & $2(0.5)$ & $37.9(9.0$ to 159.6) & $<0.001$ & $3.2(0.5$ to 19.5$)$ \\
Loss to 3-month follow-up, $\mathrm{n}(\%)$ & $13(6.5)$ & $16(4.3)$ & - & 0.253 & - \\
3-month deaths, $\mathrm{n}(\%)$ & $63(33.9)$ & $11(3.1)$ & $13.2(7.0$ to 25.1) & $<0.001$ & $1.6(0.8$ to 3.6) \\
3-month death/disability & $145(78.0)$ & $77(21.8)$ & $12.7(8.3$ to 19.5) & 0.001 & 3.3 (1.7 to 6.4) \\
\hline
\end{tabular}

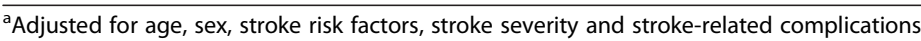

hospital, highlighting the fact that our findings should be validated in multi-site populations of Asian and nonAsian ethnicities.

Incidence of ECD was highest among the subgroup of patients with cardioembolism, and lowest among the subgroup with small-artery occlusion. These results are consistent with a study showing that altered consciousness was 3.2-fold more likely to occur in patients with cardioembolic infarction than in patients with atherothrombotic infarction [28]. In addition, the European Community Stroke Project reported that the incidence of coma and confusion within a week of onset of acute ischemic stroke was higher in the presence of atrial fibrillation than in its absence [17]. This may be because cardiac emboli are often large and therefore more likely to affect the main arteries of the cerebral vascular system, which then causes severe stroke [29].

In our cohort, age, NIHSS score on admission, massive cerebral infarction, high serum glucose on admission and history of alcohol consumption were independent risk factors of ECD. These results are consistent with a cross-sectional study of 9,044 Caucasian prehospital patients that showed that patients with abnormal GCS scores were more likely than patients with normal scores to be male and slightly older and to have a history of alcohol use, diabetes, substance abuse, stroke/transient ischemic attack and seizure [19]. Contrary to studies in French cohorts [18], our results do not support the idea that involvement of anterior circulation and multiple infarctions independently influence early conscious state in patients with acute ischemic stroke. In our cohort, CT/MRI imaging confirmed massive cerebral infarction in nearly two-thirds of patients with ECD, and $20 \%$ of these patients suffered from hemorrhagic transformation during hospitalization. And for this reason, ECD should be considered an important sign in patients with acute ischemic stroke, and it should be taken into account when designing treatment and management strategies.

Our results suggest that poor glycemic control is a predictor of ECD in diabetic patients with acute ischemic stroke. History of diabetes mellitus was identified as an independent predictor of ECD in patients with acute ischemic stroke only when the multivariate logistic regression model did not include serum glucose concentration on admission. This result may reflect the fact that hyperglycemia increases coagulation and inhibits fibrinolysis, decreasing reperfusion of ischemic tissue and increasing infarct volumes. Hyperglycemia inhibits vasodilation, increasing the production of reactive oxygen species and nicotinamide adenine dinucleotide phosphate. Hyperglycemia may also inhibit mitochondrial function in the ischemic penumbra, causing substantial intracellular acidosis [30]. Previous work has associated hyperglycemia with a higher rate of hemorrhagic complications in patients with acute ischemic stroke [30]. Our results, together with previous work, suggest that close monitoring and control of serum glucose in diabetic patients with ischemic stroke may reduce ECD incidence and improve prognosis. However, as patients in our cohort were not given hemoglobin A1c (HbA1c) detection routinely, the interpretation of our results should be cautious because we couldn't rule out the interference from stress hyperglycemia as a component of the acute phase response caused by severe stroke.

Interestingly, we found evidence that high systolic pressure on admission protects against ECD in patients with acute ischemic stroke. This may reflect auto-regulation of cerebral blood flow during the acute stage of infarction. Indeed, an earlier prospective study from our group showed that a decrease in blood pressure during the first $24 \mathrm{~h}$ after admission independently predicts one-month death in Chinese patients with acute stroke [31]. A prospective study in Spanish patients with acute ischemic stroke found that both higher and lower blood pressure during the first $24 \mathrm{~h}$ after stroke onset are associated with early neurological deterioration and poor prognosis [32]. Together, these studies highlight the importance of closely monitoring and controlling blood pressure during the first $24 \mathrm{~h}$ after stroke. In particular, they highlight the need to prevent a reduction in blood pressure in early stages of infarction. A study of 22 older American patients with delirium using 99mTc HMPAO SPECT scanning associated this conscious state with reduced blood flow in the left inferior frontal, right temporal, right occipital, and pontine regions [33]. It may be that a decrease in systemic blood pressure reduces cerebral perfusion pressure and blood flow to the ischemic penumbra, particularly in the absence of normal auto-regulation [34]. Preventing a reduction in blood pressure soon after 
infarction is consistent with official guidelines for early management of acute ischemic stroke [35].

Post-stroke complications, both neurological and medical, account for $23-50 \%$ of deaths related to acute ischemic stroke. In particular, brain edema is a major cause of death during the first week after stroke [15]. Medical and neurological complications can hinder rehabilitation, prolong hospitalization, increase healthcare costs and contribute to poor functional outcomes or death [36]. The present study associates ECD with significantly elevated risk of stroke-related complications. The most frequent neurological complication in our patients with ECD was malignant edema, followed by hemorrhagic transformation and post-stroke epilepsy. Regarding post-stroke seizures or epilepsy, the overall frequency is similar to previous reports [15]. As seizures or epilepsy is not the primary issue of the present study, patients in our cohort were not given electroencephalography routinely and we didn't record detailed information about acute symptomatic seizures which could result in early consciousness disorder. Pulmonary infection was the most common medical complication. Our results are consistent with work in US stroke patients associating greater neurological deficit with higher incidence of medical complications; indeed, that study found neurological impairment to be the strongest predictor of medical complications [37]. Since NIHSS score on admission was significantly higher among our patients with ECD than among patients without it, we speculate that more severe stroke at least partly explains the higher frequency of stroke-related complications in stroke patients with ECD.

Many complications are preventable or treatable if recognized in time. Therefore clinicians should pay close attention to patients with acute ischemic stroke and ECD in order to detect stroke-related complications as soon as possible, and thereby improve stroke outcome. Our results, and those of previous studies, highlight the need for large trials addressing the prevention and treatment of complications and their effect on stroke outcome in this stroke patient subpopulation. Indeed, the present study is one of the few to directly compare characteristics and prognosis of acute ischemic stroke patients with and without ECD. Our results suggest that the two populations differ in several clinically important respects, justifying large, well-designed studies of early management of stroke patients with ECD.

In our cohort, ECD independently predicted death during hospitalization and 3-month follow-up according to a multivariate regression model that did not take into account NIHSS score on admission or stroke-related complications. When these two parameters were included as inputs in the model, ECD was no longer an independent predictor of these outcomes, although it remained an independent predictor of 3-month death/disability. These different results echo discrepancies between previous studies showing associations of ECD with post-stroke death and poor functional outcome [38] or no such associations [39]. While these discrepancies may reflect differences in hospital populations and enrolment criteria, they are consistent with the idea that neurological deficit and stroke-related complications confound the association of ECD with death. The present study, to our knowledge, provides the first evidence that ECD elevates (3.3-fold) the risk of 3-month death/disability in patients with acute ischemic stroke, even after adjusting for confounding factors. This result is consistent with a study in French patients with acute stroke that associated acute confusional state with worse 6-month functional outcome [40].

The results of the present study should be interpreted with caution given its limitations. First, the cohort came from a single hospital, so the results may not be representative of other populations. Second, we conducted follow-up at a single time point of only 3 months, and follow-up involved a telephone interview or mailed questionnaire instead of a clinical visit, which may increase the risk of reporting bias.

\section{Conclusions}

The present study provides clear evidence that at least in Chinese populations, ECD may occur in as many as one-third of patients with acute ischemic stroke, particularly those with cardio-embolism stroke. Our analysis identifies age, stroke severity, and massive cerebral infarction as potential risk factors of ECD in ischemic stroke patients. Although ECD appears to affect anterior circulation much more often than posterior circulation, involvement of anterior circulation does not appear to independently predict ECD in patients with acute ischemic stroke. ECD in such patients is associated with malignant edema, hemorrhagic transformation and epilepsy, as well as with death and disability within 3 months after stroke onset.

\section{Acknowledgements \\ Not applicable.}

\section{Funding}

This study was sponsored by the National Natural Science Foundation of China (81371282 and 81400964)

\section{Availability of data and materials}

All relevant data are included in the manuscript. The individual data collected for each participant in our study cannot be provided in order to protect the participants' identity.

\section{Authors' contributions}

$J$, DW - collected, analyzed and interpreted the data, as well as drafted the manuscript. WT, WD, JY, and JZ - participated in study conception and design, and in data collection and interpretation. ML - contributed substantially to study conception and design, data interpretation and manuscript writing All authors critically revised the manuscript for important intellectual 
content. All the authors have read and approved the final manuscript. JL and DW contributed equally to this study.

\section{Competing interests}

The authors declare that they have no competing interests.

\section{Consent for publication}

Not applicable.

\section{Ethics approval and consent to participate}

This study was approved by the biomedical ethics committee of West China Hospital (reference number 2015300).

This study was sponsored by the National Natural Science Foundation of China. Written informed consent was obtained from participants or their guardians.

\section{Author details}

'Department of Neurology, Stroke Clinical Research Unit, West China Hospital, Sichuan University, No. 37, GuoXue Xiang, Chengdu 610041, Sichuan, People's Republic of China. 'Department of Neurology, People's Hospital of Deyang City, Deyang, People's Republic of China. ${ }^{3}$ Department of Neurology, Nanjing First Hospital, Nanjing Medical University, Nanjing, People's Republic of China.

\section{Received: 1 April 2016 Accepted: 9 August 2016}

\section{Published online: 17 August 2016}

\section{References}

1. Liu M, Wu B, Wang WZ, Lee LM, Zhang SH, Kong LZ. Stroke in China: epidemiology, prevention, and management strategies. Lancet Neurol. 2007;6(5):456-64.

2. Wong J, Traub SJ, Macnow L, Kulchycki LK. Altered Mental Status. J Emerg Med. 2008;35(4):445-8

3. Lai PF, Yiang GT, Tsai MJ, Hu SC. Analysis of Patients with Altered Mental Status in an Emergency Department of Eastern Taiwan. Tzu Chi Med J. 2009:21(2):151-5.

4. Johnston KC, Li JY, Lyden PD, Hanson SK, Feasby TE, Adams RJ, Faught Jr RE, Haley Jr EC. Medical and neurological complications of ischemic stroke: experience from the RANTTAS trial. Stroke. 1998;29(2):447-53.

5. Kumral E, Ozkaya B, Sagduyu A, Sirin H, Vardarli E, Pehlivan M. The Ege Stroke Registry: a hospital-based study in the Aegean region, Izmir, Turkey. Analysis of 2,000 stroke patients. Cerebrovasc Dis (Basel, Switzerland). 1998;8(5):278-88.

6. Vemmos KN, Takis CE, Georgilis K, Zakopoulos NA, Lekakis JP, Papamichael CM, Zis VP, Stamatelopoulos S. The Athens stroke registry: results of a five-year hospital-based study. Cerebrovasc Dis. 2000;10(2):133-41.

7. Mohr JP, Caplan LR, Melski JW, Goldstein RJ, Duncan GW, Kistler JP, Pessin MS, Bleich HL. The Harvard cooperative stroke registry. Neurology. 1978;28(8):754-62.

8. Bogousslavsky J, Van Melle G, Regli F. The Lausanne Stroke Registry: analysis of 1,000 consecutive patients with first stroke. Stroke. 1988;19(9):1083-92.

9. Foulkes MA, Wolf PA, Price TR, Mohr JP, Hier DB. The Stroke Data Bank: design, methods, and baseline characteristics. Stroke. 1988;19(5):547-54.

10. McManus J, Pathansali R, Hassan H, Ouldred E, Cooper D, Stewart R, Macdonald A, Jackson S. The course of delirium in acute stroke. Age Ageing. 2009;38(4):385

11. McManus J, Pathansali R, Stewart R, Macdonald A, Jackson S. Delirium post-stroke. Age Ageing. 2007;36(6):613-8.

12. Katzan IL, Cebul RD, Husak SH, Dawson NV, Baker DW. The effect of pneumonia on mortality among patients hospitalized for acute stroke. Neurology. 2003:60(4):620-5.

13. Karepov VG, Gur AY, Bova I, Aronovich BD, Bornstein NM. Stroke-in-evolution: infarct-inherent mechanisms versus systemic causes. Cerebrovasc Dis. 2005;21(1-2):42-6.

14. Chamorro A, Urra X, Planas AM. Infection after acute ischemic stroke: a manifestation of brain-induced immunodepression. Stroke. 2007;38(3):1097-103.

15. Balami JS, Chen RL, Grunwald IQ, Buchan AM. Neurological complications of acute ischaemic stroke. Lancet Neurol. 2011:10:357-71.

16. Di Carlo A, Lamassa M, Baldereschi M, Pracucci G, Basile AM, Wolfe CDA, Giroud M, Rudd A, Ghetti A, Inzitari D. Sex differences in the clinical presentation, resource use, and 3-month outcome of acute stroke in Europe: data from a multicenter multinational hospital-based registry. Stroke. 2003;34(5):1114-9.
17. Lamassa M, Di Carlo A, Pracucci G, Basile AM, Trefoloni G, Vanni P, Spolveri S, Baruffi MC, Landini G, Ghetti A. Characteristics, outcome, and care of stroke associated with atrial fibrillation in Europe: data from a multicenter multinational hospital-based registry (The European Community Stroke Project). Stroke. 2001;32(2):392-8.

18. Moulin T, Tatu L, Vuillier F, Berger E, Chavot D, Rumbach L. Role of a stroke data bank in evaluating cerebral infarction subtypes: patterns and outcome of 1,776 consecutive patients from the Besancon stroke registry. Cerebrovasc Dis. 2000:10(4):261-71.

19. Durant E, Sporer KA. Characteristics of Patients with an Abnormal Glasgow Coma Scale in the Prehospital Setting. West J Emerg Med. 2011:12(1):30-6.

20. Kong FY, Tao WD, Hao ZL, Liu M. Predictors of one-year disability and death in Chinese hospitalized women after ischemic stroke. Cerebrovasc Dis. 2009;29(3):255-62.

21. Damasio H. A computed tomographic guide to the identification of cerebral vascular territories. Arch Neurol. 1983;40(3):138-42.

22. Tatu L, Moulin T, Bogousslavsky J, Duvernoy H. Arterial territories of the human brain.cerebral hemispheres. Neurology. 1998;50(6):1699-708.

23. Vahedi K, Vicaut E, Mateo J, Kurtz A, Orabi M, Guichard JP, Boutron C, Couvreur G, Rouanet F, Touze E. Sequential-design, multicenter, randomized, controlled trial of early decompressive craniectomy in malignant middle cerebral artery infarction (DECIMAL Trial). Stroke. 2007:38(9):2506-17.

24. Roh JK, Kang DW, Lee SH, Yoon BW, Chang KH. Significance of acute multiple brain infarction on diffusion-weighted imaging. Stroke. 2000;31(3):688-94.

25. Adams Jr HP, Bendixen BH, Kappelle L, Biller J, Love B, Gordon D, Marsh E. Classification of subtype of acute ischemic stroke. Definitions for use in a multicenter clinical trial. TOAST. Trial of Org 10172 in Acute Stroke Treatment. Stroke. 1993;24(1):35-41.

26. Ropper AH, Samuels MA. Adams and victor's principles of neurology, ninth edition, ninth edition edn. New York: McGraw-Hill professional; 2009.

27. Van Swieten J, Koudstaal P, Visser M, Schouten H, Van Gijn J. Interobserver agreement for the assessment of handicap in stroke patients. Stroke. 1988:19(5):604-7.

28. Timsit SG, Sacco RL, Mohr JP, Foulkes MA, Tatemichi TK, Wolf PA, Price TR, Hier DB. Early clinical differentiation of cerebral infarction from severe atherosclerotic stenosis and cardioembolism. Stroke. 1992;23(4):486-91.

29. Arboix A, Alioc J. Cardioembolic Stroke: Clinical Features, Specific Cardiac Disorders and Prognosis. Curr Cardiol Rev. 2010;6(3):150-61.

30. Kruyt ND, Biessels GJ, DeVries $J H$, Roos YB. Hyperglycemia in acute ischemic stroke: pathophysiology and clinical management. Nat Rev Neurol. 2010;6:145-55.

31. Tan Y, Liu M, Wang Q. Blood pressure and prognosis in patients with acute stroke. Chin J Neurol. 2006;39(1):10-5.

32. Castillo J, Leira R, Garcia MM. Blood pressure decrease during the acute phase of ischemic stroke is associated with brain injury and poor stroke outcome. Stroke. 2004;35:52-27.

33. Fong TG, Bogardus ST, Daftary A, Auerbach E, Blumenfeld H, Modur S, Leo-Summers L, Seibyl J, Inouye SK. Cerebral perfusion changes in older delirious patients using 99mTC HMPAO SPECT. J Gerontol Series A. 2006;61(12):1294-9.

34. Johnston KC, Mayer SA. Blood pressure reduction in ischemic stroke: a two-edged sword? Neurology. 2003;2003(61):1030-1.

35. Jauch EC, Saver JL, Adams HP, Bruno A, Demaerschalk BM, Khatri P, McMullan PW, Qureshi Al, Rosenfield K, Scott PA. Guidelines for the early management of patients with acute ischemic stroke a guideline for healthcare professionals from the American Heart Association/American Stroke Association. Stroke. 2013:44(3):870-947.

36. Kumar S, Selim MH, Caplan LR. Medical complications after stroke. Lancet Neurol. 2010:9(1):105-18

37. Roth EJ, Lovell L, Harvey RL, Heinemann AW, Semik P, Diaz S. Incidence of and risk factors for medical complications during stroke rehabilitation. Stroke. 2001;32(2):523-9.

38. Kwakkel G, Wagenaar RC, Kollen BJ, Lankhorst GJ. Predicting disability in stroke: a critical review of the literature. Age Ageing. 1996;25(6):479-89.

39. Chandra RV, Law CP, Yan B, Dowling RJ, Mitchell PJ. Glasgow Coma Scale Does Not Predict Outcome Post-Intra-Arterial Treatment for Basilar Artery Thrombosis. Am J Neuroradiol. 2011;32(3):576

40. Henon H, Lebert F, Durieu I, Godefroy O, Lucas C, Pasquier F, Leys D. Confusional state in stroke: relation to preexisting dementia, patient characteristics, and outcome. Stroke. 1999;30(4):773-9. 